\title{
Shear Bands and the Development of the Brass Annealing Texture
}

\section{T. ÖZTÜRK}

Department of Metallurgical Engineering, Middle East Technical University, Ankara, Turkey

(Received March 8, 1983)

According to oriented-growth theory ${ }^{1,2}$ (Beck et al., 1953; Schmidt and Lucke, 1979) recrystallization textures are determined by the parent deformation textures through a maximum growth rate relationship. On this basis the mode of prior deformation, then, should be important only in regard to the type of deformation texture it produces. On the other hand, according to oriented-nucleation theory (Burgers and Louwerse, 1931; Dillamore and Katoh 1974) which asserts that the formation of recrystallization textures is controlled by the particularities of the deformed microstructure, the mode of prior deformation is of primary importance. In considering the rolling of metals it is usual to approximate the deformation mode as plane-strain compression, which corresponds to a pure shear deformation. Detailed analyses have been reported which characterize the deformed microstructure developed in pure shear with the aim of identifying orientations likely to develop on subsequent recrystallization (Dillamore and Katoh, 1974; Dillamore et al., 1972).

Pure shear is produced by the operation of suitably disposed positive and negative simple shears. However, the shear planes are not necessarily those of maximum shear stress (Dillamore et al., 1979) i.e. planes inclined at $45^{\circ}$ to the compression plane. In heavily rolled metals these planes are inclined at angles close to $35^{\circ}$ to the rolling plane (Duggan 
et al., 1978). While pure shear can be retained if the simple shears operate homogeneously the development of an instability, as usually occurs at high strains, causes the localization of the simple shears into separate shear bands (Dillamore et al., 1979; Duggan et al., 1978). In such cases the metal experiences simple shear locally, even though the macroscopic shape change is compatible with pure shear.

Shear bands, i.e. regions of localized shear, have been reported in the structure of many heavily rolled metals (see Duggan et al., 1978). In high stacking fault energy (SFE) f.c.c. metals, they are weak in intensity and usually occupy little volume. Low SFE metals, e.g. brass, on the other hand, develop intense shear bands when rolled to intermediate to heavy reductions. Then, the bands can make up a considerable fraction of the total volume of material (Fargette and Whitwham, 1976). Under these conditions it is doubtful whether pure shear is an appropriate description of the rolling deformation. In this note evidence is presented to show that as far as the development of the subsequent annealing texture is concerned, the deformation is better treated as simple shear (although the ideal description should involve a mixture of both simple shear and pure shear).

Figure 1(a), which is taken from a study by Zaalberg et al., (1978) shows the $\{111\}$ pole figure of an f.c.c. metal (copper) after simple shear deformation obtained by wear testing and subsequent recrystallization. Figure $1(b)$ is a $\{111\}$ pole figure typical of heavily-rolled and recrystallized brass. A close similarity between the textures depicted by the pole figures is apparent. One appears to be related to the other by a rotation about the transverse direction. As shown in Figure 1(c) a rotation of about $25^{\circ}$ appears to be sufficient to bring the pole figures into coincidence.

If the annealing texture of the rolled brass arises from recrystallization after simple shear deformation, the texture should be related to that of the worn and recrystallized material by a rotation of about $35^{\circ}$ around the transverse direction since this is the angle at which shear bands occur in the rolled metal. The angle observed here is of similar magnitude. The difference probably originates from experimental errors involved in locating precisely the shear plane and direction in the worn copper specimen.

The observed correlation between the two textures, thus, suggests that the brass annealing texture originates from recrystallization within shear bands. A similar suggestion was made by Noda et al. (1978), on the basis of correlation they observed between the orientation distribution 
Burgers, W. G. and Louwerse, P. C. Z. Physik, 61, 605 (1931).

Dillamore, I. L. and Bush, A. C. Textures of Materials Vol. 1 (Eds. C. Gottstein and K Lucke) Berlin, Springer-Verlag, 367 (1978).

Dillamore, I. L. and Katoh, H. Metal Science J., 8, 73 (1974).

Dillamore, I. L., Morris, P. L., Smith, C. J. E. and Hutchinson, W. B. Proc. Roy. Soc., A329, 405 (1972).

Dillamore, I. L., Roberts, J. G. and Bush, A. C. Metal Science J., 13, 73 (1969).

Duggan, B. T., Hatherly, M., Hutchinson, W. B. and Wakefield, P. T. Metal Science J., 12, 343 (1978).

Fargette, B. and Whitwham, D. Mem. Sci. Rev. Met., 73, 197 (1976).

Noda, T., Plege, B. and Grewen, J. Textures of Materials, Vol 1 (Eds. G. Gottstein and K. Lucke) Berlin, Springer-Verlag, 443 (1978).

Öztürk, T. Ph.D thesis, University of Cambridge (1978).

Schmidt, U. and Lucke, K. Text. Cryst. Solids, 3, 85 (1979).

Zaalberg, R. J., Dautzenberg, J. H. and Klostermann, J. A. Textures of Materials Vol. 1 (Eds. G. Gottstein and K. Lucke) Berlin, Springer-Verlag, 379 (1978). 